\title{
An accelerated multistart derivative-free framework for the beam angle optimization problem in IMRT
}

\author{
Humberto Rocha ${ }^{1,2}$, Joana M. Dias ${ }^{1,2}$, Tiago Ventura ${ }^{3}$, \\ Brígida C. Ferreira ${ }^{4}$, and Maria do Carmo Lopes $^{3}$ \\ 1 Faculdade de Economia, Universidade de Coimbra, \\ 3004-512 Coimbra, Portugal \\ 2 INESC-Coimbra, \\ 3030-290 Coimbra, Portugal \\ 3 Serviço de Física Médica, IPOC-FG, EPE, \\ 3000-075 Coimbra, Portugal \\ 4 School for Allied Health Technologies, \\ 4400-330 Porto, Portugal \\ hrocha@mat.uc.pt, joana@fe.uc.pt, tiagoventura@ipocoimbra.min-saude.pt, \\ bcf@estsp.ipp.pt, mclopes@ipocoimbra.min-saude.pt
}

\begin{abstract}
Radiation therapy, either alone or combined with surgery or chemotherapy, is one of the main treatment modalities for cancer. Intensity-modulated radiation therapy (IMRT) is an advanced form of radiation therapy, where the patient is irradiated using non-uniform radiation fields from selected beam angle directions. The goal of IMRT is to eradicate all cancer cells by delivering a radiation dose to the tumor volume, while attempting to spare, simultaneously, the surrounding organs and tissues. Although the use of non-uniform radiation fields can favor organ sparing, the selection of appropriate irradiation beam angle directions - beam angle optimization - is the best way to enhance organ sparing. The beam angle optimization (BAO) problem is an extremely challenging continuous non-convex multi-modal optimization problem. In this study, we present a novel approach for the resolution of the BAO problem, using a multistart derivative-free framework for a more thoroughly exploration of the search space of the highly non-convex $\mathrm{BAO}$ problem. As the objective function that drives the BAO problem is expensive in terms of computational time, and a multistart approach typically implies a large number of function evaluations, an accelerated framework is explored. A clinical case of an intra-cranial tumor treated at the Portuguese Institute of Oncology of Coimbra is used to discuss the benefits of the accelerated multistart approach proposed for the BAO problem.
\end{abstract}

Keywords: IMRT, Beam angle optimization, Multistart, Derivativefree optimization 


\section{Introduction}

Radiation therapy, either alone or combined with surgery or chemotherapy, is one of the main treatment modalities for cancer. The goal of radiation therapy is to eradicate all cancer cells by delivering a radiation dose to the tumor volume, while attempting to spare, simultaneously, the surrounding organs and tissues. Radiation therapy is used with curative intent or to palliate symptoms giving important symptom relief. Intensity-modulated radiation therapy (IMRT) is an advanced form of radiation therapy, where the radiation beam is modulated by a multileaf collimator allowing its discretization into small beamlets of different intensities. This discretization of the radiation beam enables the irradiation of the patient using non-uniform radiation fields from selected beam angle directions. The use of non-uniform radiation fields in IMRT provides an accurate control of the different doses to be optimized which can favor organ sparing. However, appropriate selection of individualized irradiation beam directions - beam angle optimization (BAO) - depositing in an additive way the total radiation dose in the tumor while attempting to spare the surrounding organs and tissues that only receive radiation from a small subset of radiation beams, is the best way to enhance a proper organ sparing.

Despite the fact that for some treatment sites, in particular for intra-cranial tumors, BAO substantially improves plan quality [4], in clinical practice, coplanar equispaced beam directions, i.e. evenly spaced beam directions that lay on the plane of rotation of the linear accelerator's gantry, are still commonly used. Alternatively, beam directions are manually selected on a long trial-and-error procedure by the treatment planner, as commercial treatment planning systems have limited resources available for BAO. One of the reasons for the scarce commercial offer for beam angle directions optimal selection is the difficulty of solving the BAO problem, a highly non-convex multi-modal optimization problem on a large search space [12].

The BAO approaches can be separated into two different classes. The first class considers a discrete sample of all possible beam angle directions and addresses the $\mathrm{BAO}$ problem as a combinatorial optimization problem. As the best ensemble of beam angles cannot be obtained through exhaustive searches, in a reasonable computational time, different methods are commonly used to guide the searches including genetic algorithms [13], simulated annealing [14], particle swarm optimization [15], gradient search [12], neighborhood search [1], response surface [2], branch-and-prune [16] or hybrid approaches [5]. The combinatorial formulation of the BAO problem leads to an NP hard problem. Thus, there is no algorithm known capable of finding, in a polynomial run time, the optimal solution of the combinatorial BAO problem [3]. Another common and successful combinatorial approach is iterative $\mathrm{BAO}[3,8]$, where beams are sequentially added, one at a time, to a treatment plan, significantly reducing the number of beam combinations. The second class of $\mathrm{BAO}$ approaches considers a completely different methodological approach by exploring the continuous search space of the highly non-convex BAO problem $[18,19,20]$. 
In this study, we present a novel approach that belong to the second class of approaches for the resolution of the BAO problem. A multistart derivativefree framework is sketched for a more thoroughly exploration of the continuous search space of the highly non-convex BAO problem. As the objective function that drives the $\mathrm{BAO}$ problem is expensive in terms of computational time, and a multistart approach typically implies a large number of function evaluations, an accelerated multistart framework is tested using a clinical case of an intra-cranial tumor treated at the Portuguese Institute of Oncology of Coimbra (IPOC). The paper is organized as follows. In the next Section we describe an accelerated multistart framework for the BAO problem. Computational tests are presented in Section 3. In the last Section we have the conclusions.

\section{Multistart derivative-free framework for BAO}

\subsection{BAO formulation}

The formulation of the BAO problem as a continuous optimization problem was proposed in our previous works $[18,19,20]$. In order to model the BAO problem as a mathematical optimization problem, a measure of the quality of the beam angles ensemble is required. The straightforward measure for driving the BAO problem is the optimal solution of the fluence map optimization (FMO) problem $[1,3,8,12,13,14,16,18,19,20]$, the problem of finding the optimal radiation intensities. Let $n$ be the fixed number of (coplanar) beam directions, a continuous formulation for the BAO problem is obtained by selecting an objective function $f$ such that the best set of beam angles is obtained for the function's minimum:

$$
\begin{aligned}
& \min f\left(\theta_{1}, \ldots, \theta_{n}\right) \\
& \text { s.t. }\left(\theta_{1}, \ldots, \theta_{n}\right) \in \mathbb{R}^{n},
\end{aligned}
$$

where $\theta_{i}, i=1, \ldots, n$ are beam angles selected from all continuous beam irradiation directions. For this study, the objective function $f\left(\theta_{1}, \ldots, \theta_{n}\right)$ that measures the quality of the beam angle ensemble $\theta_{1}, \ldots, \theta_{n}$ was the FMO problem objective function, modeled as a multicriterial optimization problem. Nevertheless, as the FMO model is used as a black-box function, the conclusions drawn using this particular formulation of the FMO problem can be extended to different FMO formulations.

\subsection{FMO for an intra-cranial tumor case}

Typically, the FMO problem is modeled as a weighted sum function with conflicting objectives which difficult their trade-off. Thus, treatment plan optimization is inherently a multicriteria procedure. Different multicriteria approaches have been proposed for the FMO problem where solutions can be selected from a set of Pareto-optimal treatment plans a posteriori $[11,17]$ or a set of criteria (objectives and constraints) that have to be met is defined a priori $[6,7,8]$. The 
latter approach is more suitable for a fully automated BAO procedure. Thus, a multicriterial optimization procedure based on a prescription called wish-list $[6,7,8]$ is used to address the FMO problem.

Table 1 depicts the wish-list constructed for the clinical intra-cranial tumor case treated at IPOC. Intra-cranial cases are complex tumors to treat with radiation therapy due to the large number of sensitive organs in this region. Beyond the planning target volume (PTV), i.e. the tumor to be irradiated, a large number of organs at risk (OARs) are included in the wish-list. Different dose levels were defined for the tumor (PTV-T) and for the lymph nodes (PTV-N), according to the IPOC protocols defined for this pathology. Several auxiliary structures (PTV-T Ring, PTV-N Ring, PTV-N shell and External Ring) were constructed by computerized volume expansions to support the dose optimization.

The wish-list contains 11 hard constraints, all maximum-dose constraints, that have to be strictly met. It also contains 28 prioritized objectives that are sequentially optimized following the priorities defined in the wish-list. For the target dose optimization, the logarithmic tumor control probability (LTCP) was considered [8], LTCP $=\frac{1}{N_{T}} \sum_{l=1}^{N_{T}} e^{-\alpha\left(D_{l}-T_{l}\right)}$, where $N_{T}$ is the number of voxels (small volume elements) in the target structure, $D_{l}$ is the dose in voxel $l, T_{l}$ is the prescribed dose, and $\alpha$ is the cell sensitivity parameter. For most OARs maximum-dose constraints were considered. For some OARs, a generalized Equivalent Uniform Dose $(g E U D)$ objective was considered [8], $g E U D=$ $k\left(\frac{1}{N_{S}} \sum_{l} D_{l}^{a}\right)^{\frac{1}{a}}$, where $k$ is the number of treatment fractions, $N_{S}$ the number of voxels of the discretized structure, $D_{l}$ the dose in voxel $l$ and $a$ is the tissuespecific parameter that describes the volume effect.

A primal-dual interior-point algorithm tailored for multicriteria IMRT treatment planning, 2pєc [6], was used for optimization of the FMO problem using the described wish-list. The $2 p \epsilon c$ algorithm automatically generates a single Pareto optimal IMRT plan for a given number of beams. For a detailed description of the $2 p \epsilon c$ algorithm see Breedveld et al.[6].

\subsection{Multistart approach for the continuous BAO problem}

Multistart approaches have two phases that can be designated as global and local phases [10]. In the global phase, a number of starting points is selected for which the objective function is evaluated. Then, local search procedures are used to improve each of the starting points outcome. In previous works, we have shown that a beam angle set can be locally improved in a continuous manner using Pattern Search Methods (PSM) $[18,19,20]$. An important feature of PSM is its ability to converge globally, i.e., from arbitrary points to local minimizers [21]. Furthermore, PSM have the ability to avoid local entrapment and require few function evaluations to converge. Thus, PSM were selected for the local search procedure to be embedded in the multistart framework.

In the global phase, typically, starting points (beam ensembles) are randomly selected. However, for search spaces with peculiar characteristics as the BAO continuous search space, different strategies need to be adopted. As the order of 
Table 1. Wish-list for the intra-cranial tumor case.

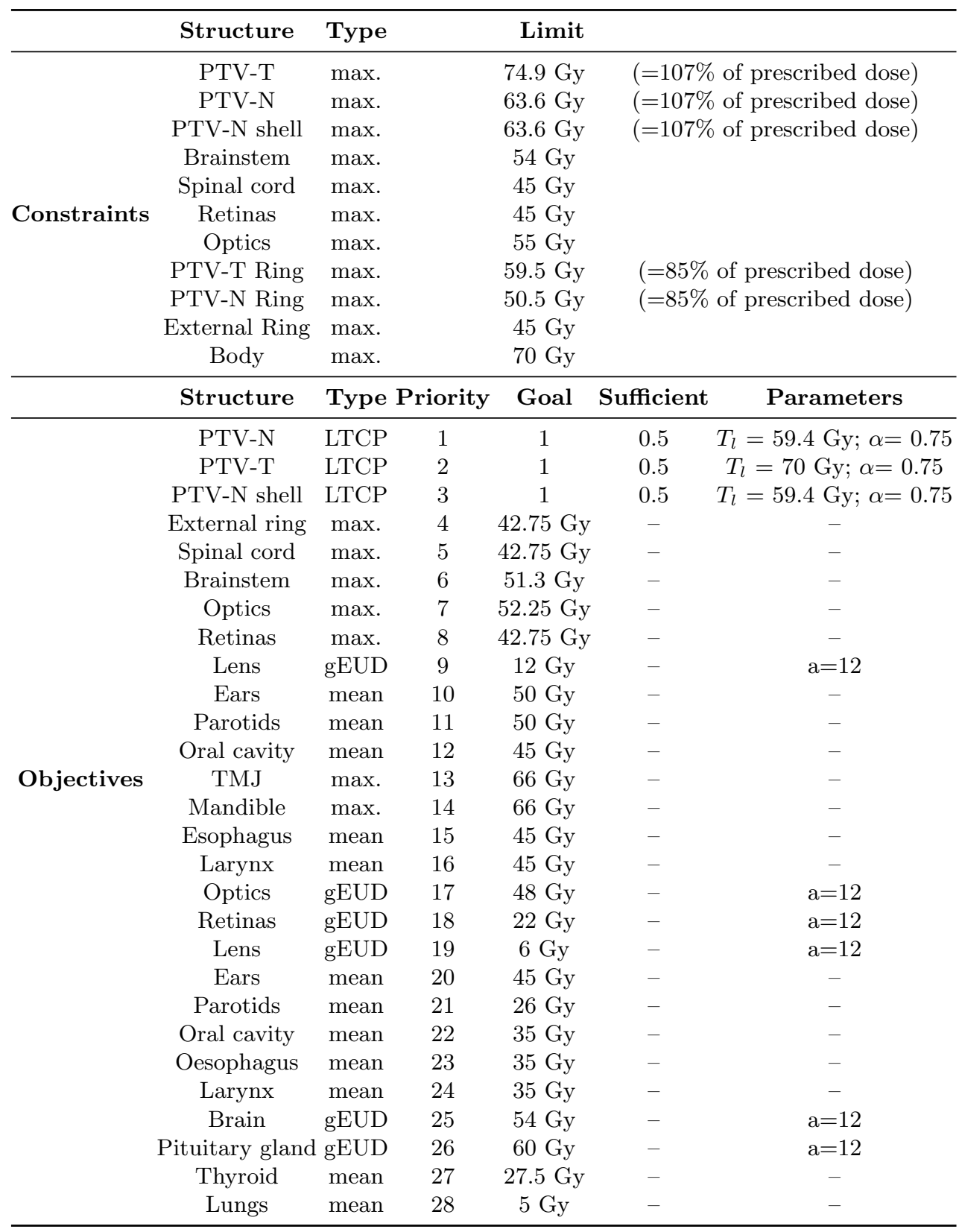


the beam directions of a beam angle ensemble is irrelevant, the BAO continuous search space has symmetry properties. This means that different solutions can in reality correspond to the same solution, the only difference being that the same angles appear in different positions. This simple observation allows for a drastic reduction of the search space by simply ordering the angles in each solution. For $n$-beam directions, by keeping the beam angles sorted, the search space is reduced by $2^{n}$. E.g., for the 7-beam angle search space, the reduced search space is only $0.78 \%$ of $[0,360]^{7}$. However, the reduced search space may take a peculiar shape. Thus, a strategy to sample the reduced search space is required.

The strategy sketched for selecting the starting points considers all the possible distributions of the sorted beam angle directions by quadrants. For illustration purposes, all possible distributions of 3-beam angle directions by the four quadrants are depicted in Fig. 1. Examples of 3-beam angle directions for each one of the 20 possible distributions by the four quadrants are displayed in Fig. 1(a) while Fig. 1(b) displays the corresponding painted cubes of the reduced search space. In general, for the $n$-beam angle direction search space, the total number of (hyper)cubes of the entire search space is $4^{n}$ while the number of (hyper)cubes of the reduced search space is the combination with repetition of $\left(\begin{array}{c}n+4-1 \\ 4\end{array}\right)=\frac{(n+4-1) !}{4 !(n-1) !}$. E.g., for the 7-beam angle optimization problem, the reduced search space has 120 (hyper)cubes while the entire search space has 16384 . A good strategy for sampling the reduced search space consists in selecting one starting point for each one of the (hyper)cubes of the reduced search space. Such strategy guarantees that the starting points belong to the reduced search space, they are well spread and most importantly they cover well all the reduced search space.

A major drawback of multistart methods, particularly for a parallel setting, is that the same region of the search space may be simultaneously searched by local procedures originated from starting points of different regions. Thus, the same local minima can be found more than once wasting precious computational time. A generalization of the notion of region of attraction of a local minimum can be used to avoid overlap of local searches and simultaneously accelerate the optimization procedure. One can define each (hyper)cube of the reduced search space as a region of attraction of a local minimum and allow a single "active" local search for each region of attraction. If two local searches end up simultaneously searching the same (hyper)cube during the optimization procedure, only the local search with the current best solution, i.e. with the solution corresponding to the lowest objective function value, will remain active which accelerates the overall optimization process.

At the end of the global phase of the multistart method, the objective function value is evaluated at the $N=\frac{(n+4-1) !}{4 !(n-1) !}$ initial beam ensembles selected, $\mathbf{x}_{i}^{0} \in[0,360]^{n}, i=1, \ldots, N$. For many of the regions, the starting points will lead to poor objective function results. E.g., beam ensembles with many (or all) beams in the same quadrant will have beams too close to produce good results. Thus, another strategy to accelerate the multistart framework is to explore only the most promising regions at each iteration. In order to do so, a boolean vec- 


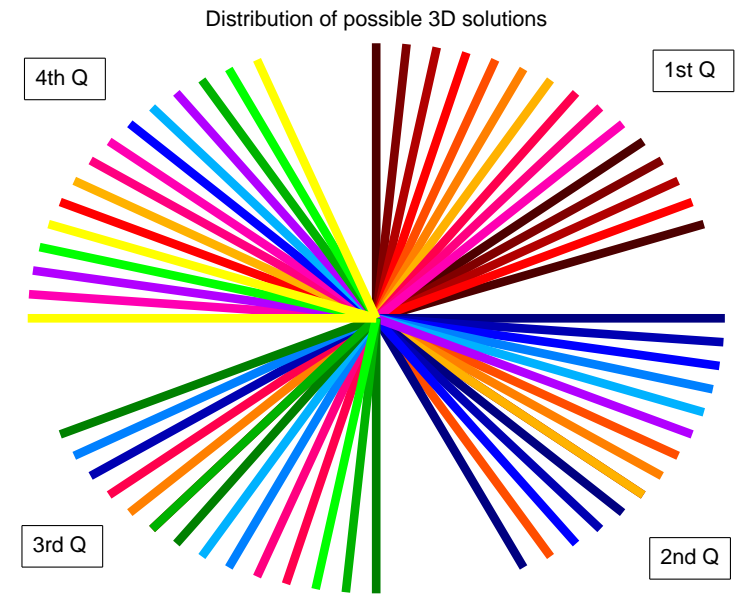

(a)

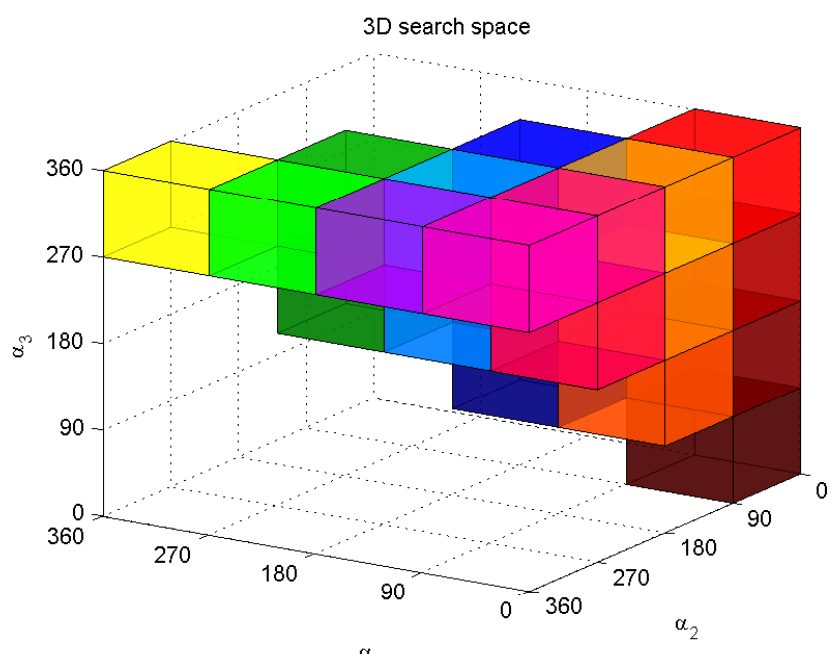

(b)

Fig. 1. Distribution of 3-beam directions by the four quadrants - 1(a) and the corresponding cubes in the search space $[0,360]^{3}-1(\mathrm{~b})$.

tor, Active $_{N \times 1}$, that stores the information of which regions have active local search procedures is updated in every iteration and the overall best objective function value $f_{*}^{k}=f\left(\mathbf{x}_{*}^{k}\right)=\min \left\{f\left(\mathbf{x}_{1}^{k}\right), f\left(\mathbf{x}_{2}^{k}\right), \ldots, f\left(\mathbf{x}_{N}^{k}\right)\right\}$ is determined at each iteration $k$. The regions $i$ where local searches remain active, $\mathbf{A c t i v e}_{i}=1$, 
correspond to the regions whose best objective function value is not worst than the overall best objective function value $f_{*}$ within a defined threshold $p \geq 0$, i.e. $f_{i} \leq(1+p) f_{*}$. The different local search procedures can always progress towards regions whose local procedures are not currently active. The accelerated multistart derivative-free algorithm is described in Algorithm 1.

\section{Algorithm 1 Accelerated multistart derivative-free algorithm framework}

\section{Initialization:}

- Choose $\mathbf{x}_{i}^{0} \in[0,360]^{n}, i=1, \ldots, N$;

- Evaluate, in parallel, the objective function value at $\mathbf{x}_{i}^{0} \in[0,360]^{n}, i=1, \ldots, N$;

- Set $\mathbf{x}_{i}^{\text {best }} \leftarrow \mathbf{x}_{i}^{0}, i=1, \ldots, N$ and $f_{i}^{b e s t} \leftarrow f\left(\mathbf{x}_{i}^{0}\right), i=1, \ldots, N$;

- Determine the best initial beam ensemble $\mathbf{x}_{*}^{0}$ and the corresponding best initial objective function value $f_{*} \leftarrow f\left(\mathbf{x}_{*}^{0}\right)$;

- Choose $p \geq 0$. Set Active A $_{i} \leftarrow 1$ if $f_{i}^{0} \leq(1+p) f_{*}^{0}$, Active A $_{i} \leftarrow 0$ otherwise;

- Set $k \leftarrow 1$;

- Choose a positive spanning set, a step size $s_{i}^{1}>0, i=1, \ldots, N$ and a minimum step size $s_{\text {min }}$ for PSM algorithm;

\section{Iteration:}

1. Using PSM, perform local search, in parallel, for the active regions;

2. For each active region $i$ do

If local search is successful, i.e. $f\left(\mathbf{x}_{i}^{k+1}\right)<f\left(\mathbf{x}_{i}^{k}\right)$ then

$$
\begin{aligned}
& \text { If } \mathbf{x}_{i}^{k+1} \text { remains in region } i \text { then } \\
& \mathbf{x}_{i}^{\text {best }} \leftarrow \mathbf{x}_{i}^{k+1} ; \\
& f_{i}^{\text {best }} \leftarrow f\left(\mathbf{x}_{i}^{k+1}\right) ; \\
& \text { Else }
\end{aligned}
$$

Active $_{i} \leftarrow 0$

Find $j \neq i$ where $\mathbf{x}_{i}^{k+1}$ is;

If $f\left(\mathbf{x}_{i}^{k+1}\right)<f\left(\mathbf{x}_{j}^{b e s t}\right)$ then

$$
\begin{aligned}
\mathbf{x}_{j}^{\text {best }} & \leftarrow \mathbf{x}_{i}^{k+1} ; \\
f_{j}^{b e s t} & \leftarrow f\left(\mathbf{x}_{i}^{k+1}\right) ;
\end{aligned}
$$

Active $_{j} \leftarrow 1$;

Else

$$
\begin{aligned}
& s_{i}^{k+1} \leftarrow \frac{s_{i}^{k}}{2} \\
& \text { If } s_{i}^{k+1}<s_{\min } \text { then } \\
& \text { Active } \mathbf{e}_{i} \leftarrow 0 ;
\end{aligned}
$$

3. Determine the overall best beam ensemble $\mathbf{x}_{*}^{k}$ and the corresponding overall best objective function value $f_{*} \leftarrow f\left(\mathbf{x}_{*}^{k}\right)$;

4. Set Active A $_{i} \leftarrow 1$ if $f_{i}^{k} \leq(1+p) f_{*}^{k}$, Active $_{i} \leftarrow 0$ otherwise;

5. Set $k \leftarrow k+1$. If any region is still active then return to step 1 for a new iteration. 


\section{Computational results}

A modern 8-core workstation was used to perform the computational tests. An in-house optimization platform written in MATLAB, named YARTOS, developed at Erasmus MC Cancer Institute in Rotterdam [6,7,8], was used to import DICOM images, create new structures, compute dosimetric input, compute the optimal fluence dose maps and compute/visualize dosimetric output. The YARTOS fluence map optimizer, $2 p \epsilon c$, was used to obtain the optimal value of the FMO problem required to drive our multistart derivative-free BAO framework. As the FMO model is treated as a black-box function, other FMO models can be easily coupled with this multistart BAO framework.

The PSM algorithm implemented for the local search procedure of the multistart derivative-free framework used the maximal positive basis $([I-I])$, where $I$ is the $n$-dimensional identity matrix. These directions correspond to the rotation of each beam direction clockwise and counter-clockwise for a certain amount (step-size) at each iteration. The initial step-size considered was $s^{1}=2^{5}=32$ and the minimal value allowed was one $s_{\min }=1$, defining the stopping criteria. As the step-size is halved at unsuccessful iterations, this choice of initial stepsize implies that all beam directions will be integer until the termination criteria, when the step-size becomes inferior to one. No trial point was computed in the search step to avoid increasing the number of FMO evaluations.

Treatment plans with 7-beam angle directions, obtained using the multistart derivative-free framework and denoted Multistart BAO, were compared against treatment plans with 7-beam angle directions, obtained using iterative $\mathrm{BAO}$ and denoted Iterative $B A O$. These treatment plans were compared against treatment plans with 7-beam angle equispaced coplanar ensembles, denoted Equi, commonly used at IPOC and in clinical practice to treat intra-cranial tumor cases [1] and used here as benchmark.

The performance of a BAO algorithm should be evaluated using two criteria. While the main goal is to obtain the best objective function value possible, another important goal is to obtain a good solution as fast as possible. For the $\mathrm{BAO}$ problem, the computation of the optimal value of the FMO problem dominates the computational time, consuming more than $95 \%$ of the overall computational time. In our accelerated multistart framework, the total number of FMO evaluations depends on the threshold $p$ that defines which regions have active local search procedures. Obviously, by decreasing $p$ the number of function evaluations will decrease. The main goal of this study is to acknowledge how much can we decrease that parameter, accelerating the multistart strategy, without significantly deteriorating the objective function value. We tested $p=1, p=0.5, p=0.1, p=0.05$ and $p=0.01$ corresponding to consider, at each iteration, all regions active, regions for which the best objective function value is not $50 \%(10 \%, 5 \%$ or $1 \%$, respectively) worst than the overall best objective function value. The results of these tests are displayed in Fig. 2. In Fig. 2(a) the relative FMO improvement comparing the benchmark beam ensemble, Equi, (0\% improvement) and the best treatment plan, Multistart BAO with $p=1$, (100\% improvement) is displayed. The number of FMO evaluations required to 


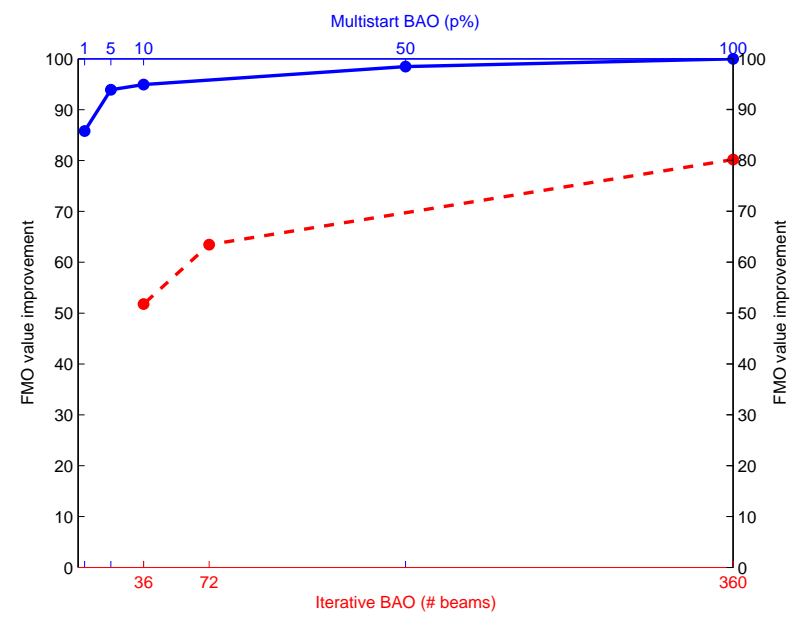

(a)

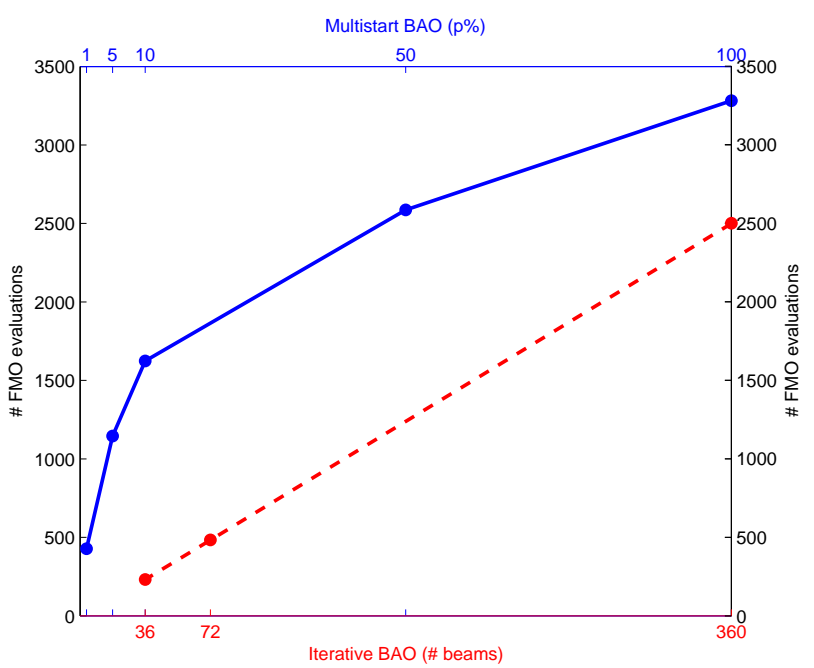

(b)

Fig. 2. Relative FMO value improvement - 2(a) and corresponding number of function evaluations $-2(\mathrm{~b})$.

obtain the corresponding solutions are displayed in Fig. 2(b). It can be seen that the results in terms of objective function value slowly deteriorate until $p=0.1$. For smaller values of $p$ the deterioration of the optimal FMO value is more accentuated. A steep decrease on the number of FMO evaluations is seen as the value of $p$ decreases. E.g., the number of function evaluations for $p=0.1$ is infe- 
rior to half of the number of function evaluations for $p=1$ at a very small cost of objective function deterioration.

Iterative $B A O$ results for different discrete samples of all possible beam irradiation directions are also depicted in Fig. 2. Gantry angles are typically discretized into equally spaced beam directions with a given angle increment. We considered angle increments of 10, 5 and 1 degrees, originating 3 discrete samples with 36,72 and 360 beam angles. The iterative BAO greedy strategy drastically reduces the number of FMO problem evaluations compared to the combinatorial $\mathrm{BAO}$, particularly for larger angle increments. However, since the search space is truncated at each iteration, at the very first iteration this strategy can disregard the best ensembles with $n$-beam directions. In Fig. 2(a) the consequences of that greedy strategy are clearly seen with worse FMO improvements even for larger numbers of FMO evaluations (for 360 beam angles).

In clinical practice, the quality of the results is typically assessed by cumulative dose-volume histograms (DVHs). The DVH displays the fraction of a structure's volume that receives at least a given dose. DVH results for Multistart $B A O$ with $p=0.1$, Iterative $B A O$ with 360 angles and Equi treatment plans are displayed in Fig. 3. For clarity, DVH curves were divided into two figures and only spinal cord, brainstem, oral cavity, parotids and tumor volumes are displayed. The DVH curves show that for a similar tumor coverage, a better organ sparing is generically obtained by the treatment plans using the optimized beam directions, in particular by Multistart BAO treatment plans. In Fig. 3(a), Multistart BAO DVH curves show a better sparing of spinal cord and oral cavity compared to Iterative $B A O \mathrm{DVH}$ curves, corresponding to better maximum and mean doses for spinal cord and oral cavity (26.1 Gy vs 28.7 Gy and 33.6 Gy vs $34.3 \mathrm{~Gy}$, respectively). At IPOC, two of the salivary glands, submandibular glands and sublingual glands, are included in the oral cavity structure. These two salivary glands, along with parotids, are very important in saliva production. Thus, the enhanced oral cavity sparing is of the utmost interest to prevent xerostomia caused by over-irradiation of salivary glands. In Fig. 3(b), both Multistart $B A O$ and Iterative $B A O$ DVH curves show a better sparing of brainstem compared to Equi DVH curves, corresponding to an improvement of 3 Gy on the brainstem maximum dose. The significant sparing obtained for brainstem is very important, e.g. for re-irradiation cases. Parotids show similar results for all treatment plans.

\section{Conclusions and Future Work}

Multistart methods with local search procedures are globally convergent [9] and their interest and application fields continue to rise [10]. For an extremely challenging non-convex optimization problem as the BAO problem, a multistart derivative-free framework is a suitable approach. This approach combines a global strategy for sampling the search space with a local strategy for improving the sampled solutions. While the local strategy using PSM proved, in previous works, to be successful in improving locally beam angle ensembles, requiring few 


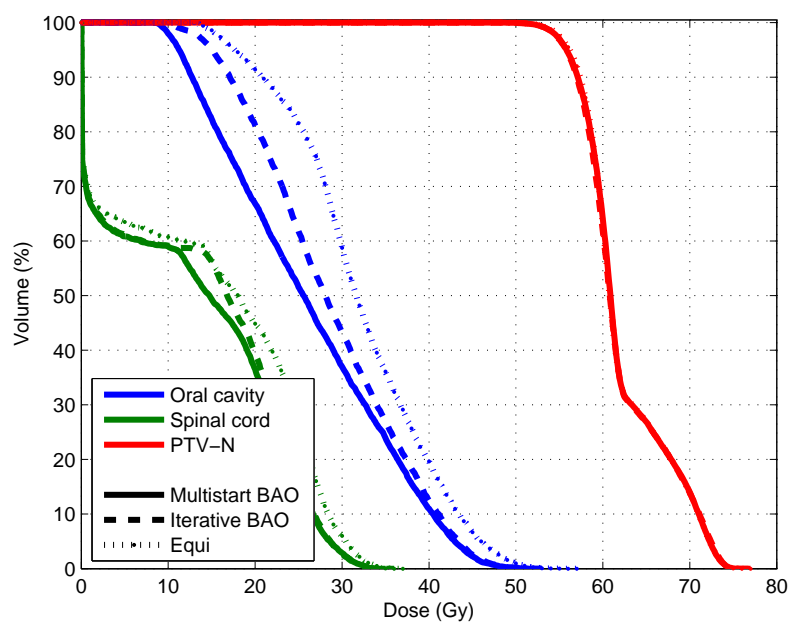

(a)

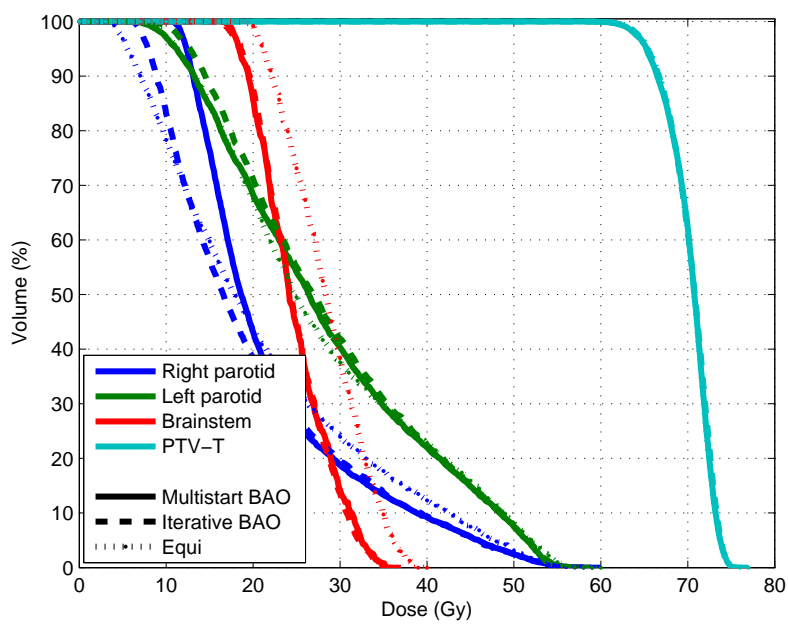

(b)

Fig. 3. Cumulative dose volume histogram comparing the results obtained by Multistart $B A O$ with $p=0.1$, Iterative $B A O$ with 360 angles and Equi treatment plans.

function evaluations and avoiding local entrapment, the global strategy inherently requires a larger number of function evaluations. Several strategies were embedded in this methodology to reduce, as much as possible, the number of function evaluations, including the non-random strategy sketched to take advantage of a reduced search space and the use of a generalization of regions of 
attraction of a local minimum. Knowing in advance that some regions would most probably produce poor solutions, a strategy to explore locally the most promising regions at each iteration was also drafted and tested in this study.

The accelerated multistart derivative-free framework was tested using a clinical intra-cranial tumor treated at IPOC to acknowledge how much can we speed up the optimization process before significant deterioration of results occur. For the intra-cranial clinical case retrospectively tested, FMO optimal values of treatment plans obtained using Multistart BAO beam directions suffer small deterioration if up to $10 \%$ of the most promising regions where locally explored at each iteration corresponding to a decrease superior to $50 \%$ on the number of function evaluations. Thus, for the accelerated multistart derivative-free framework, the results indicate that a good compromise between the number of function evaluations and the quality of the solutions is the threshold $p=0.1$. Furthermore, treatment plans obtained using Multistart $B A O$ beam directions clearly outperform results obtained by Iterative $B A O$ treatment plans even when the number of function evaluations was large (360 beam angle set).

As future work, different strategies for accelerating the proposed multistart derivative-free framework will be tested including the truncation of the number of iterations of the FMO. The multistart framework presented is tailored for coplanar beam directions and in future work this framework will be extended to include noncoplanar beam directions as well.

\section{Acknowledgements}

This work has been supported by the Fundação para a Ciência e a Tecnologia (FCT) under project grant UID/MULTI/00308/2013. We would like to show gratitude to Ben Heijmen and Sebastiaan Breedveld for giving us permission to install Yartos.

\section{References}

1. Aleman, D.M., Kumar, A., Ahuja, R.K., Romeijn, H.E., Dempsey, J.F.: Neighborhood search approaches to beam orientation optimization in intensity modulated radiation therapy treatment planning. J. Global Optim. 42, 587-607 (2008)

2. Aleman, D.M., Romeijn, H.E., Dempsey, J.F.: A response surface approach to beam orientation optimization in intensity modulated radiation therapy treatment planning. INFORMS J. Comput.: Computat. Biol. Med. Appl. 21, 62-76 (2009)

3. Bangert, M., Ziegenhein, P., Oelfke, U.: Characterizing the combinatorial beam angle selection problem. Phys. Med. Biol. 57, 6707-6723 (2012)

4. Bangert, M., Ziegenhein, P., Oelfke, U.: Comparison of beam angle selection strategies for intracranial imrt. Med. Phys. 40011716 (2013)

5. Bertsimas, D., Cacchiani, V., Craft, D., Nohadani, O.: A hybrid approach to beam angle optimization in intensity-modulated radiation therapy. Comput. Oper. Res. 40, 2187-2197 (2013)

6. Breedveld, S., Storchi, P., Keijzer, M., Heemink, A.W., Heijmen, B.: A novel approach to multi-criteria inverse planning for IMRT. Phys. Med. Biol. 52, 6339-6353 (2007) 
7. Breedveld, S., Storchi, P., Heijmen, B.: The equivalence of multicriteria methods for radiotherapy plan optimization. Phys. Med. Biol. 54, 7199-7209 (2009)

8. Breedveld, S., Storchi, P., Voet, P., Heijmen, B.: iCycle: integrated, multicriterial beam angle, and profile optimization for generation of coplanar and noncoplanar IMRT plans. Med. Phys. 39, 951-963 (2012)

9. Brimberg, J., Hansen, P., Mladenovic, N.: Convergence of variable neighborhood search. Les Chaiers du Gerard (2004).

10. Mart, R., Resende, M.G.C., Ribeiro, C.C.: multistart methods for combinatorial optimization. Eur. J. Oper. Res. 226 1-8 (2013)

11. Craft, D., Halabi, T., Shih, H., Bortfeld, T.: Approximating convex Pareto surfaces in multiobjective radiotherapy planning. Med. Phys. 33, 3399-3407 (2006)

12. Craft, D.: Local beam angle optimization with linear programming and gradient search. Phys. Med. Biol. 52, 127-135 (2007)

13. Dias, J., Rocha, H., Ferreira, B.C., Lopes, M.C.: A genetic algorithm with neural network fitness function evaluation for IMRT beam angle optimization. Cent. Eur. J. Oper. Res. 22, 431-455 (2014)

14. Dias, J., Rocha, H., Ferreira, B.C., Lopes, M.C.: Simulated annealing applied to IMRT beam angle optimization: A computational study. Physica Medica 31, 747$756(2015)$

15. Li, Y., Yao, D., Yao, J., Chen, W.: A particle swarm optimization algorithm for beam angle selection in intensity modulated radiotherapy planning. Phys. Med. Biol. 50, 3491-3514 (2005)

16. Lim, G.J., Cao, W.: A two-phase method for selecting IMRT treatment beam angles: Branch-and-Prune and local neighborhood search. Eur. J. Oper. Res. 217, 609-618 (2012)

17. Monz, M., Kufer, K.H., Bortfeld, T.R., Thieke, C.: Pareto navigation Algorithmic foundation of interactive multi-criteria IMRT planning. Phys. Med. Biol. 53, 985998 (2008)

18. Rocha, H., Dias. J., Ferreira, B.C., Lopes, M.C.: Selection of intensity modulated radiation therapy treatment beam directions using radial basis functions within a pattern search methods framework. J. Glob. Optim. 57 1065-89 (2013)

19. Rocha, H., Dias. J., Ferreira, B.C., Lopes, M.C.: Beam angle optimization for intensity-modulated radiation therapy using a guided pattern search method. Phys. Med. Biol. 58 2939-53 (2013)

20. Rocha, H., Dias, J., Ferreira, B.C., Lopes, M.C.: Pattern search methods framework for beam angle optimization in radiotherapy design. Appl. Math. Comput. 219, $10853-10865$ (2013)

21. Torczon, V.: On the convergence of pattern search algorithms. SIAM J. Optim. 7, $1-25(1997)$ 